\title{
THE KERNEL OF THE POINCARÉ SERIES OPERATOR
}

\author{
THOMAS A. METZGER
}

\begin{abstract}
By modifying a proof of Ljan, a natural basis for the kernel of the Poincaré series operator in the Bers space can be given. The basic idea behind the proof also extends to give such a basis in the case of a general Kleinian group and a discontinuous group acting on certain domains in $\mathbf{C}^{\boldsymbol{n}}$.
\end{abstract}

1. Introduction. Let $\Gamma$ be a Fuchsian group acting on the unit disk $D$ in the complex plane. Let $q$ be an integer, $q>2$, and suppose that $f$ is a holomorphic function defined on $D$. The Poincare series for $f$ with respect to $\Gamma$ of weight $q$ is defined to be

$$
\theta_{q}(f, \Gamma)(z)=\sum_{A \in \Gamma} f(A z) A^{\prime}(z)^{q},
$$

whenever this series converges independently of the particular arrangement chosen. It is clear that whenever the series in (1) exists it defines a holomorphic function on $D$ which is an automorphic form of weight $q$ with respect to $\Gamma$, i.e., it is a holomorphic function $F$ such that

$$
F[A(z)] A^{\prime}(z)^{q}=F(z) \text { for all } A \text { in } \Gamma \text { and } z \text { in } D .
$$

The series in (1) were originally considered by Poincare [8] and as he recognized there is seemingly no way to assert, apriori, that $\theta_{q}(f, \Gamma)(z) \neq 0$. Thus Poincaré considered meromorphic functions $f$ and defined (1) for this latter case. Recently, Ljan [6] considered the problem of finding the kernel of this operator in a natural space of functions, the Bers space $A_{q}(D)$. He proved that a certain set was dense in $A_{q}(D) \cap \operatorname{Ker} \theta_{q}$. Unfortunately, it is very difficult to compute any of the functions which belong to this set. This is due to the fact that his functions are defined by an integral representation. In this note it is shown that, by modifying the proof of Ljan, one can get a natural set with the property that its closed linear span in $A_{q}(D)$ equals $\operatorname{Ker} \theta_{q} \cap$ $A_{q}(D)$. This set has the advantage that, given the group $\Gamma$, each individual function in the set is easily computed. Of course, this does not solve the classical Poincaré problem as it does not yield a decision process for determining $\operatorname{Ker} \boldsymbol{\theta}_{\boldsymbol{q}}$.

2. Preliminaries. The Bers space $A_{q}(\Gamma)$ is defined to be the Banach space of holomorphic automorphic forms of weight $q$ (i.e., (2) holds) such that

$$
\iint_{\Omega}|f(z)|\left(1-|z|^{2}\right)^{q-2} d x d y<\infty
$$

Received by the editors October 28, 1977 and, in revised form, October 10, 1978.

AMS (MOS) subject classifications (1970). Primary 30A58; Secondary 30A46, 10D10, 10D20. 
where $\Omega$ is a fundamental region for $\Gamma$ with the property that the two dimensional measure of the boundary of $\Omega$ is zero. $A_{q}$ (id) is denoted by $A_{q}(D)$. These spaces were introduced by Bers (see [1]) and he proved:

TheOREM A (Bers [2]). If $f$ is in $A_{q}(D)$ then $\theta_{q}(f, \Gamma)$ exists. Moreover, $\theta_{q}$ : $A_{q}(D) \rightarrow A_{q}(\Gamma)$ is a bounded linear operator of norm less than or equal to one which is onto.

The Banach space $B_{q}(T)$ is defined to be the space of holomorphic automorphic forms of weight $q$ such that

$$
\sup _{D}|f(z)|\left(1-|z|^{2}\right)^{q}<\infty .
$$

This space is related to $A_{q}(\Gamma)$ by:

ThEOREM B (BERs [2]). The dual space of $A_{q}(\Gamma)$ is $B_{q}(\Gamma)$ under the Petersson inner product

$$
(F, G)=\iint_{\Omega} F(z) \overline{G(z)}\left(1-|z|^{2}\right)^{2 q-2} d x d y
$$

In order to motivate our result we define

$$
K=\operatorname{Ker} \theta_{q} \cap A_{q}(D) \text {. }
$$

It is clear that $K$ is a closed subspace of $A_{q}(D)$. The following computation involving a switch of summation and integration is standard (see [1] and [7]). If $f$ is in $K$, then (1) is identically zero and Theorem B implies that, for every $G$ in $B_{q}(\Gamma)$,

$$
\begin{aligned}
0 & =\iint_{\Omega} \theta_{q}(f, \Gamma)(z) \overline{G(z)}\left(1-|z|^{2}\right)^{2 q-2} d x d y \\
& =\sum_{A \in \Gamma} \iint_{\Omega} f(A z) A^{\prime}(z)^{q} \overline{G(z)}\left(1-|z|^{2}\right)^{2 q-2} d x d y \\
& =\sum_{A \in \Gamma} \iint_{A \Omega} f(z) \overline{G(z)}\left(1-|z|^{2}\right)^{2 q-2} d x d y \\
& =\iint_{D} f(z) \overline{G(z)}\left(1-|z|^{2}\right)^{2 q-2} d x d y .
\end{aligned}
$$

The switch of summation and integration is justified by the fact that the integrals converge absolutely. Thus we have shown:

LEMMA 1. $f$ is in $K$ if and only if $(f, G)=0$ for all $G$ in $B_{q}(\Gamma)$.

Note. It makes sense to consider $(f, G)$ for $f$ in $A_{q}(D)$ and $G$ in $B_{q}(\Gamma)$ since $B_{q}(\Gamma) \subseteq B_{q}(D) \equiv B_{q}(\mathrm{id})$, for all groups $\Gamma$.

Define $\kappa$ to be the (closed) subspace of $A_{q}(D)$ spanned by $\{g(z ; k, A)$ : $A \in \Gamma$ and $k=0,1,2, \ldots\}$ where

$$
g(z ; k, A)=z^{k}-(A z)^{k} A^{\prime}(z)^{q} .
$$


THEOREM 1. $\kappa=K$.

3. Proof of Theorem 1. It is well known that if $f$ is in $A_{q}(D)$ and $g(z)=f(z)-f(A z) A^{\prime}(z)^{q}$ for some $A$ in $\Gamma$ then, $\theta_{q}(g, \Gamma)(z) \equiv 0$. It follows immediately that $\kappa \subseteq K$. To prove the opposite containment we first prove:

Proposition 2. Suppose that $h$ is in $B_{q}(D)$ and that

$$
(g(\cdot ; k, A), h)=0
$$

for all $A$ in $\Gamma$ and $k=0,1,2, \ldots$ Then $h$ is in $B_{q}(\Gamma)$.

Proof. If $h(z)=\sum_{n=0}^{\infty} b_{n} z^{n}$, then an elementary computation yields $\left(z^{k}, h\right)$ $=\pi \bar{b}_{k} B(2 q-1, k+1)$ where $B(p, q)$ is the usual beta function. Similarly, if $A$ is in $\Gamma$ and

$$
h(N(z)) N^{\prime}(z)^{q}=\sum_{n=0}^{\infty} c_{n} z^{n},
$$

with $N=A^{-1}$, an elementary change of variables in (3) yields that

$$
\left((A(z))^{k}\left(A^{\prime}(z)\right)^{q}, h\right)=\pi \bar{c}_{k} B(2 q-1, k+1) .
$$

Thus, (6) implies that (2) holds for all $A$ in $\Gamma$ and the proof is complete.

To complete the proof of the theorem assume that $\kappa$ is strictly contained in $K$ and let $l$ be a bounded linear functional on $K$ which is not identically zero on $K$ and which annihilates $\kappa$. By the Hahn-Banach theorem $l$ can be extended to a bounded linear functional on $A_{q}(D)$, which is also denoted by $l$. Theorem B implies that $l(f)=(f, h)$ for some $h$ in $B_{q}(D)$. Since $l$ annihilates $\kappa$ it follows from Proposition 1 that $h$ belongs to $B_{q}(\Gamma)$. It follows from Lemma 1 that $(f, h)=0$ for all $f$ in $K$ and then $l \equiv 0$. This contradiction completes the proof.

4. Concluding remarks. It follows immediately from the definition of $\kappa$ that:

Corollary $1 . \operatorname{dim} K=\infty$.

Finally we note that the proof given above can be generalized to almost every situation of current interest. The reason for this is that the basic ingredient is Proposition 1, and if one can suitably modify the functions in (5) so that this proposition still holds then the theorem will follow with the same proof. We shall indicate below what the correct modification should be in the case $\Gamma$ is a Kleinian group or a discrete group acting on certain domains in $\mathbf{C}^{n}$.

Let $\Gamma$ be a Kleinian group with region of discontinuity $\Omega$. It is well known that $A_{q}(\Omega)$ has a Bergman kernel function $K_{q}(z, \zeta)$ which has the property that $\left(K_{q}(\cdot, \zeta), g\right)=\overline{g(\zeta)}$ for all $g$ in $B_{q}(\Omega)$. Let $\zeta_{k}$ be a dense countable subset of $\Omega$ and substitute $K_{q}\left(z, \zeta_{k}\right)$ for $z^{k}$ in (5). It is clear that Proposition 1 remains valid and thus Theorem 1 holds with $\kappa$ being the linear subspace of $A_{q}(\Omega)$ spanned by these new functions (see Kra [5] for complete details).

A similar observation can be made in the case of several complex variables. 
In [4], Earle described a class of domains in $\mathbf{C}^{n}$ for which analogous results to those of Bers for one variable could be proved. The domains again have a Bergman kernel function so that upon taking a dense countable subset and using the same substitution as above one sees immediately that Theorem 1 holds in this case.

It should also be noted that the same idea works in the case of relative Poincaré series. In [3], Drasin defined such series and proved that the needed results from the Bers space theory still hold. It is not hard to see that a proper substitute in this case for the functions $z^{k}$ in (5) is $\theta\left(K_{q}\left(\cdot, \zeta_{k}\right), \Gamma_{1}\right)(z)$ where $\left\{\zeta_{k}\right\}$ are a dense subset of $D / \Gamma_{1}$ and $\Gamma_{1}$ is a subgroup of the group $\Gamma$.

Unfortunately, in all three of the cases above one loses the explicitness of (5). This is due to the fact that one merely knows of the existence of the Bergman kernel but one does not have, in the general case, an explicit function.

It should also be noted that the main theorem above does not solve the classical problem of Poincaré. Obviously, one cannot decide via some algorithm whether or not a specific function belongs to $K$. Finally, we note that the set $\kappa$ is not well behaved under auxiliary conjugation of the group.

Note (ADDED IN PROOF). J. Elstrodt (private communication) has pointed out that one need only consider $A$ in a generating set in definition (5) and Theorem 1 remains valid. The proof is exactly the same as the one above.

\section{REFERENCES}

1. L. Bers, Completeness theorems for Poincare series in one variable, Proc. of Internat. Sympos. on Linear Spaces (Jerusalem, 1960), Jerusalem Academic Press, Jerusalem, 1961, pp. 88-100.

2. _ Automorphic forms and Poincaré series for infinitely generated Fuchsian groups, Amer. J. Math. 87 (1965), 196-214.

3. D. Drasin, Cusp forms and Poincaré series, Amer. J. Math. 90 (1968), 356-365.

4. C. J. Earle, Some remarks on Poincaré series, Compositio Math. 21 (1969), 167-176.

5. I. Kra, Automorphic forms and Kleinian groups, Benjamin, Reading, Mass., 1972.

6. G. M. Ljan, On the kernel of Poincaré's $\theta$ operator, Soviet Math. Dokl. 17 (1976), 1283-1285.

7. H. Petersson, Über Weierstrasspuntke und die expliziten Darstellung der automorphen Formen von reeler Dimension, Math. Z. 52 (1949), 32-59.

8. H. Poincaré, Mémoire sur les fonctions fuchsiennes, Acta Math. 1 (1882), 193-294.

Department of Mathematics, University of Pittsburgh, Pittsburgh, Pennsylvania 15260 